\title{
A PROBLEMÁTICA DO ENSINO DE RADIAÇÃO NO ENSINO MÉDIO: UM ESTUDO DE CASO.
}

\author{
Matheus Carlos da Silva ${ }^{1}$ \\ Priscila Freitas-Lemes ${ }^{2}$ \\ Douglas Carlos Vilela ${ }^{3}$ \\ José Silvério Edmundo Germano ${ }^{4}$ \\ Inácio Malmonge Martin ${ }^{5}$
}

Resumo: A propagação de energia de um ponto a outro é chamada de Radiação, conceito físico empregado em muitas situações do nosso dia-a-dia. Sabe-se que apesar desse ser um importante tópico da física, é um dos menos explorados pelos professores ao longo do Ensino Médio, seja pela falta de tempo ou pela formação deficiente dos professores. Com a finalidade de entender como está sendo tratado o assunto Radiação, o objetivo deste trabalho é quantificar o nível de conhecimento dos alunos de ensino médio no tema radiação. Para isso, realizou-se um estudo de caso na escola Anglo-TAUBATÉ, com 224 alunos do terceiro ano e cursinho pré-vestibular, através da aplicação de um questionário e de aulas supervisadas. Os resultados apontam os pontos de melhor conhecimento dos alunos e também os mais deficientes dessa relação ensino-aprendizagem. A fim de melhorar o ensino deste tópico, está sendo desenvolvido um simulador, que poderá auxiliar o ensino.

Palavras-chave: Radiação; Ensino Médio; Simulador.

\footnotetext{
${ }^{1}$ Mestrando em Ensino de Física/Instituto Tecnológico de Aeronáutica, Brasil. E-mail: matheus92carlosdasilva@hotmail.com.

${ }^{2}$ Pós-Doutoranda em Ensino de Física/Instituto Tecnológico de Aeronáutica, Brasil. E-mail: piprisc@gmail.com ${ }^{3}$ Doutorando em Ensino de Física/Instituto Tecnológico de Aeronáutica, Brasil. E-mail: douglascarlosvilela@gmail.com.

${ }^{4}$ Professor Doutor/Instituto Tecnológico de Aeronáutica, Brasil. E-mail: silverio@ita.br.

${ }^{5}$ Professor Doutor/Instituto Tecnológico de Aeronáutica, Brasil. E-mail: martin@ita.br.
} 